\title{
Digitization of dental alginate impression: Three-dimensional evaluation of point cloud
}

\author{
So-Ri KIM¹, Wan-Sun LEE ${ }^{1}$, Woong-Chul KIM¹, Hea-Young KIM² and Ji-Hwan KIM¹ \\ ${ }^{1}$ Department of Dental Laboratory Science and Engineering, College of Health Science, Korea University, 161, Jeongneung-ro, Seongbuk-gu, Seoul \\ 136-712, Korea \\ ${ }^{2}$ Department of Public Health Science, Graduate School \& BK21+ Program in Public Health Science, Korea University, 161, Jeongneung-ro, \\ Seongbuk-gu, Seoul 136-712, Korea \\ Corresponding author, Ji-Hwan KIM; E-mail: kjh2804@korea.ac.kr
}

\begin{abstract}
The purpose of this study was to evaluate the digitization of alginate impressions by analyzing differences between the scan data of two types of impressions (alginate and rubber) taken from the master die and the scan data for the master die. The master die and impressions were digitized using a dental laser scanner (7 series, Dental Wings, Montreal, Canada). The crown portion of the abutment teeth in the digital data of 20 impressions was divided into three regions: cervical surface, middle surface, and occlusal surface. An independent $t$-test showed a significant difference $(p<0.05)$ in the mean difference for each experimental group (alginate and rubber). One-way ANOVA and Tukey's honest significant difference test revealed a significant difference $(p<0.05)$ among the three regions in the rubber impression. The results of this study also carefully suggest the possibility of digitization of alginate impressions in the future.
\end{abstract}

Keywords: Laser scanner, Impression scanning, Computer analysis, Stereolithography, Point cloud

\section{INTRODUCTION}

Dental impression materials are classified according to the setting mechanism and purpose of use; their function is to record the spatial relationship of oral tissues in a precise manner. Historically, impressions were taken using non-elastic materials for both soft and hard tissues $^{1}$. Alginate was originally introduced to replace non-elastic materials, particularly for impressions of hard tissues ${ }^{2}$.

In general, rubber impressions are used for the production of a stone model (type IV) for fixed dental prostheses. In contrast, irreversible alginate impressions are predominately used to produce a stone model (type III) for removable dental prostheses ${ }^{3}$. Alginate impression materials are economical compared with polyester, and additive and condensation silicone. In addition, the use of alginate impression material has several advantages such as ease of use, the ability to obtain a relatively precise impression, and the lack of requirement for specific equipment ${ }^{4)}$. However, in alginate impressions, absorbed water may shrink or expand upon the continuous reaction of sol, and water evaporation can occur ${ }^{3)}$. The absorption and evaporation of water results in an imprecise dental model; therefore, alginate impression material is used less frequently for the production of conventional fixed dental prostheses in which the prosthesis is manufactured following the production of a stone model (type IV).

The introduction of the computer-aided design/ computer-aided manufacture (CAD/CAM) system to

Color figures can be viewed in the online issue, which is available at J-STAGE.

Received Nov 14, 2014: Accepted Jun 12, 2015

doi:10.4012/dmj.2014-313 JOI JST.JSTAGE/dmj/2014-313 dentistry in the 1980's resulted in the application of laser scanning technology for the production of dental prostheses ${ }^{5}$. While the early system could scan dental models, scanning of the dental impression only became possible as new technologies developed. Currently, rubber impression digitization and scanning models are commonly used ${ }^{6,7)}$. Furthermore, digitization of alginate impressions is now possible. Precise impression taking is crucial for enhancing the suitability of the final prosthesis when using both the production method for dental prostheses with $\mathrm{CAD} / \mathrm{CAM}$ and the conventional method of prostheses production ${ }^{8)}$. In order to evaluate the degree of precision for such impressions, software enabling an extremely precise comparison and analysis, even for non-regular and geometric shapes such as those of the teeth, was recently introduced ${ }^{9}$. Furthermore, the validity of a system that analyzes the 3-dimensional (3D) surface model, developed by the utilization of laser scanning, has also been reported ${ }^{10)}$.

The majority of previous studies on the digitization of impressions have focused on rubber impressions ${ }^{11-14}$; relatively few studies have scanned the alginate impressions used frequently in current dental practice. Therefore, this study aimed to evaluate the digitization of alginate impressions by analyzing differences in scan data between two types of impressions (alginate and rubber) taken from the master die and the scan data for the master die.

\section{MATERIALS AND METHODS}

Tooth preparation and production of master dies

The maxillary right first molar of a full mouth dental 
model (AG-3, Frasaco, Tettnang, Germany) composed of synthetic resins was selected. The abutment teeth were prepared as follows: amount of occlusal reduction, 1.5 $\mathrm{mm}$; chamfer margin, $1.2 \mathrm{~mm}$ and $360^{\circ}$; and axial wall angle, $5^{\circ}$. Ten silicone (Dublisil, Dreve Dentamid, Unna, Germany) molds were produced for the preparation of the master dies using acrylic abutment teeth. A type IV dental stone (Fujirock EP, GC, Leuven, Belgium) was poured into the silicone molds as a vacuum mixture, according to the manufacturer's instructions. From this, 10 master dies were produced (Fig. 1).

\section{Preparation of the impression}

A custom tray was produced by attaching a handle (length, $50 \mathrm{~mm}$; width, $15 \mathrm{~mm}$ ) to a plastic cup (diameter, $23 \mathrm{~mm}$; height, $20 \mathrm{~mm}$ ) with small holes, and this was used to take precise impressions. A total of 20 impressions were prepared: 10 rubber impressions (Aquasil Ultra XLV, Dentsply, Germany) and 10 alginate impressions (Aroma Fine Plus Normal SET, GC, Tokyo, Japan). The master dies were removed from the impression according to the setting time of each impression material, as specified by the manufacturer.

\section{Digitization of the master dies and impressions}

The advantage of the laser scanner (7 series, Dental Wings) used in this study is that it provides scan data with high precision through primary basic scanning and secondary detailed scanning. Furthermore, the MultiDie Scanning Software module allows one-time scanning through the arrangement of 30 dies in the multi-die tool.

Scanning of alginate impressions was simultaneously performed during impression taking, while the scanning of the rubber impressions was conducted 1 day after their production. A 3-D digital model (Stereolithography file: STL file) was obtained by arranging 10 master dies into the multi-die tool. Scanning spray (Powder Scan Spray, VITA, USA) was spread onto two impressions

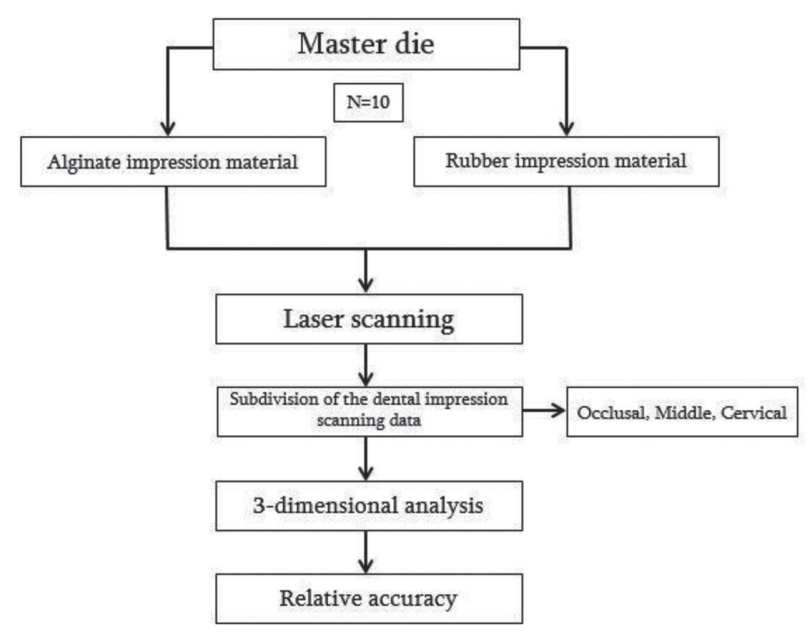

Fig. 1 Schematic study workflow chart. for effective digitization. The scanning of 10 rubber impressions and 10 alginate impressions was followed by the fixation of the custom tray onto the impression scanning holder. At this point, the impression scan mode was selected in the software.

\section{Analysis of digitized impressions}

The 3-D digital models (STL file) of the 10 master dies were defined as the CAD reference model (CRM), while the digital models (point cloud, action script communication [ASC] file) of the two types of impressions were defined as the rubber impression (RI) experimental group and the alginate impression (AI) experimental group. The unnecessary portion below the margin on the obtained 3-D virtual model was deleted using modeling software (PowerSHAPE Pro 2014, Delcam plc, Birmingham, UK) in order to enable rapid data analysis, precise results, and simplification of data size.

Ten master casts were produced and numbered from 1 to 10 . Following this, the scan data of the ten master casts of abutment teeth were obtained and utilized as CRM. Scan data of the ten impressions were also acquired and the data of the master casts and rubber and alginate impressions, with corresponding numbers, were compared from 1 to 10 using CRMs, in order to analyze relative accuracy.

For precise analysis, 3-D surface models of the impressions were segmented into three portions (CS: cervical surface, MS: middle surface, and OS: occlusal surface) and data portioning was performed using modeling software (PowerSHAPE Pro 2014, Delcam plc). The digital model for the impression, composed of 60 data points in total, was converted into an ASC file using specific software (CopyCAD, Delcam plc). The ASC file format is a convenient method to read, write, and express point data when only 3-D points are used in 3-D geometry applications. As a 3-D laser scanner, the point cloud model performs basic print-outs. The 3-D coordinate file is the most comprehensive point cloud file format and ASCII is the format type for this file. ASC is equivalent to ASCII and its file extension is defined as

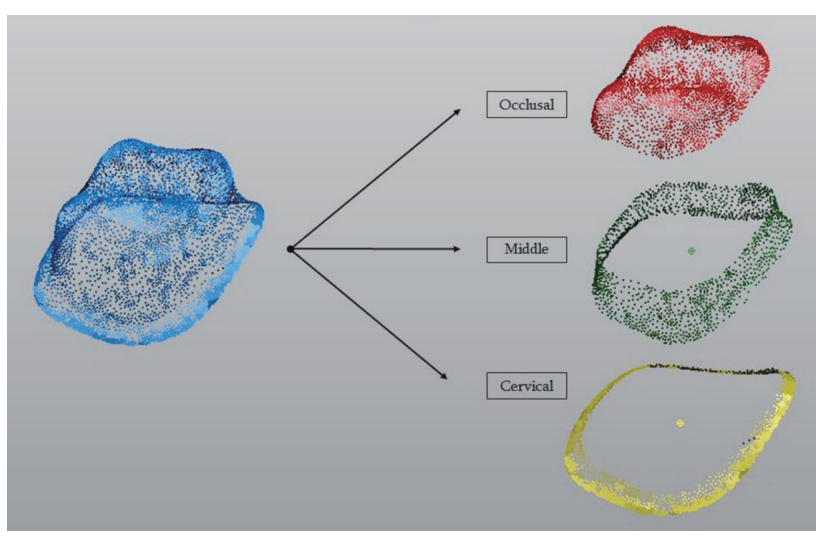

Fig. 2 Subdivisions of the dental impression scanning data. 
'.asc'. The ASC files were projected onto the 3-D surface models of the master dies and the differences between the two models were evaluated with inspection-software (PowerINSPECT, Delcam plc) (Fig. 2).

\section{Statistical analysis}

The statistical significance of the measured results was assessed using a statistics program (SPSS Statistics 20.0, IBM, Chicago, IL, USA). The independent $t$-test was utilized to assess the significance of the differences in mean values between the two experimental groups (AI and RI). The one-way ANOVA test was used to verify the differences between the three regions (CS, MS, and OS) in each experimental group. Tukey's multiple comparison test was performed to compare the statistical differences within each group. Statistical significance was accepted at $p<0.05$.

\section{RESULTS}

The difference in point cloud of the experiment group when compared with CRM for rubber impressions and alginate impressions was $-3.0 \pm 3.2 \mu \mathrm{m}$ and $9.8 \pm 6.9 \mu \mathrm{m}$, respectively. Results of independent sample $t$-tests showed a significant difference in the mean values of both groups $(p<0.05)$ (Table 1$)$.

Hence, the results can be interpreted as follows: (1) digital data of rubber impressions differed less with the master pattern than it did with the alginate impressions; (2) rubber impressions decreased in size by an average of $3 \mu \mathrm{m}$ when compared with the master pattern, as indicated by the negative values (Fig. 3); and (3) alginate impressions increased in size by an average of $9.8 \mu \mathrm{m}$ when compared with the master pattern, as indicated by the positive values (Fig. 4).

One-way ANOVA was conducted in order to evaluate the differences between regions in each impression. Additionally, Tukey's multiple comparison test was conducted to compare differences in CS, MS, and OS; results revealed that rubber impressions did not present a significant difference between OS and MS, while there were significant differences observed between OS and $\mathrm{CS}$ and between MS and SC $(p<0.05)$. By contrast, there were no significant differences observed for alginate impressions (Table 2).

Both Table 2 and Table 3 show results from within-

Table 1 Discrepancies between the groups from the alignments between the master dies (CRM) and the impressions (point cloud) (unit $=\mu \mathrm{m})$

\begin{tabular}{|c|c|c|c|c|c|c|c|c|c|}
\hline \multirow{2}{*}{ Part } & \multicolumn{4}{|c|}{ Rubber impression (RI) } & \multicolumn{5}{|c|}{ Alginate impression (AI) } \\
\hline & Mean & $\mathrm{SD}$ & Max. & Min. & Mean & $\mathrm{SD}$ & Max. & Min. & $p$-value \\
\hline Entire & -3.00 & 3.23 & 1 & -10 & 9.80 & 6.97 & 24 & 3 & 0.027 \\
\hline OS & -3.60 & 1.07 & -2 & -6 & 4.90 & 4.31 & 13 & -1 & 0.007 \\
\hline MS & -3.40 & 2.12 & -1 & -8 & 6.30 & 4.40 & 12 & -3 & 0.084 \\
\hline $\mathrm{CS}$ & -1.00 & 1.41 & 1 & -4 & 3.80 & 2.86 & 11 & 1 & 0.208 \\
\hline
\end{tabular}

Table 2 Discrepancies within the groups from the alignments between the master dies (CRM) and the impressions (point cloud) (unit $=\mu \mathrm{m})$

\begin{tabular}{cccc}
\hline Material & OS & MS & CS \\
\hline Rubber impression & $-3.60^{\mathrm{a} \#}$ & $-3.40^{\mathrm{a}}$ & $-1.00^{\mathrm{b}}$ \\
Alginate impression & 4.90 & 6.30 & 3.80 \\
\hline
\end{tabular}

\#Different letters indicate significant differences according to Tukey's HSD test $(p<0.05)$.

Table 3 Average number of points and dimensional changes in the point clouds of each group

\begin{tabular}{|c|c|c|c|c|}
\hline \multirow{2}{*}{ Part } & \multicolumn{2}{|c|}{ Rubber impression } & \multicolumn{2}{|c|}{ Alginate impression } \\
\hline & Number of nodes & Dimensional change (\%) & Number of nodes & Dimensional change (\%) \\
\hline Entire & 7290 & \multirow{3}{*}{-0.60} & 7929 & \multirow{4}{*}{0.55} \\
\hline OS & 2330 & & 2588 & \\
\hline MS & 1417 & & 1533 & \\
\hline $\mathrm{CS}$ & 3543 & & 3809 & \\
\hline
\end{tabular}


group comparisons conducted in both rubber and alginate impressions. Achievement of a greater number of points and a more precise mean value in the $\mathrm{CS}$ region can be explained by these results; as indicated by the numerical values, we were able to perform more precise work in this region compared to other regions since the most lateral location is where light contact is most convenient during the scanning process.

\section{DISCUSSION}

One of the strengths of impression scanning is that the provision of a stone model is unnecessary for the preparation of dental prostheses. In addition, the digital data permits simple data storage and performance of data searches, as well as smooth communication for prostheses preparation. Furthermore, such data aids dentists in the planning and evaluation of treatment, and

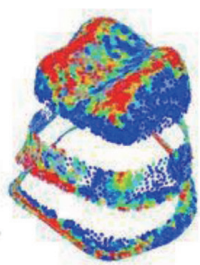

(a)

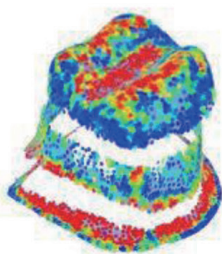

$(f)$

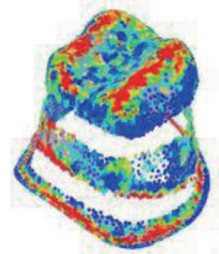

(b)

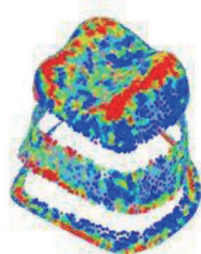

(c)

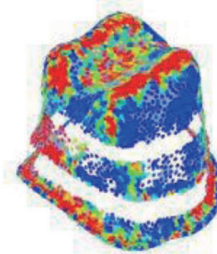

(d)

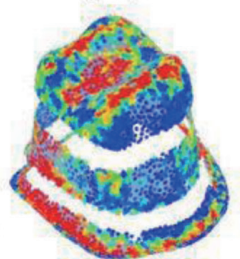

(g)

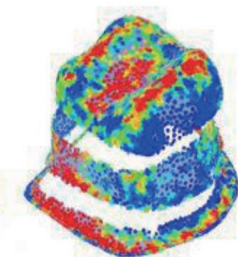

(h)

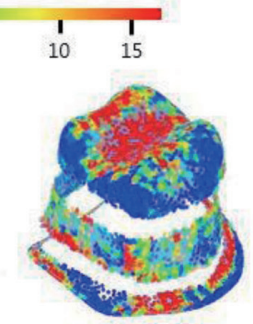

(i)

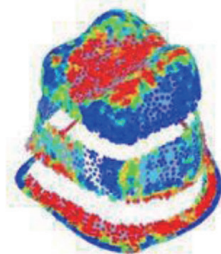

(e)

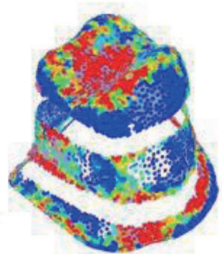

(j)

Fig. 3 Color difference map of the discrepancy distribution of the molar showing the fit alignment between the master dies and the rubber impression $(\mathrm{a}-\mathrm{j})$.

In the color difference map, a positive value (red) indicates that the master die is larger than the compared rubber impression and a negative value (blue) indicates that the master die is smaller than the compared rubber impression.

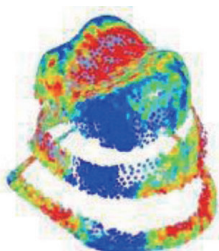

(a)

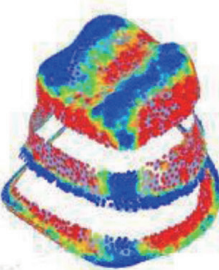

(f)

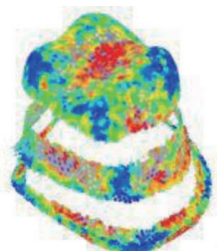

(b)

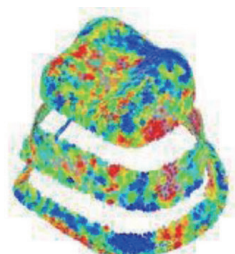

(c)

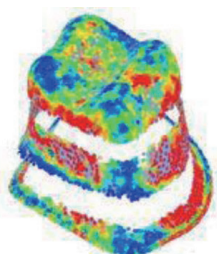

(d)
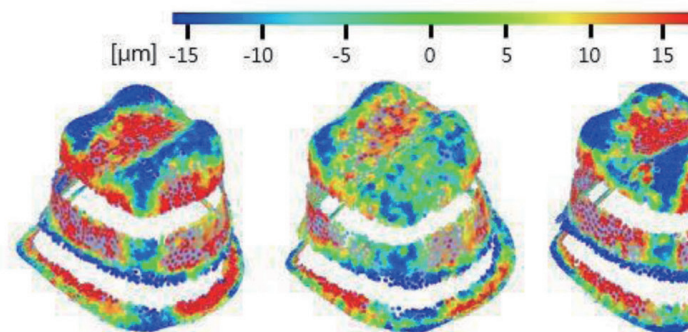

(g)

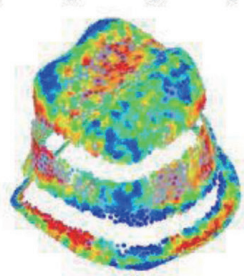

(h)

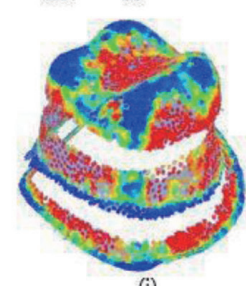

(i)

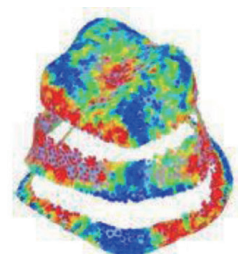

(e)

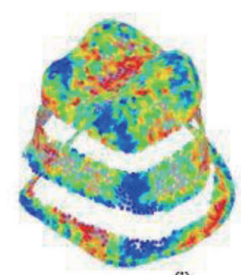

(j)

Fig. 4 Color difference map of the discrepancy distribution of the molar showing the fit alignment between the master dies and the alginate impression $(a-j)$.

In the color difference map, a positive value (red) indicates that the master die is larger than the compared alginate impression and a negative value (blue) indicates that the master die is smaller than the compared alginate impression. 
improves treatment outcome ${ }^{15)}$. 3-D superimpositional software, which enables an extremely precise comparison and analysis of irregular and geometric shapes, has been utilized to evaluate such data ${ }^{9,12,16-19)}$. The analysis software used in this study (PowerINSPECT) allows measurements to be obtained on STL files and triangle models, used in each part and product, as well as rapid prototyping.

In this study, a negative value $(-3.00 \pm 3.2 \mu \mathrm{m})$ for the silicon rubber impressions and a positive value $(9.80 \pm 6.9 \mu \mathrm{m})$ for the alginate impressions was recorded, indicating that more shrinkage occurred in the silicon rubber impressions compared with the master dies, and that expansion occurred in the alginate impressions. In addition, comparison of the numerical values revealed that the rubber impressions reproduced the master dies in a more precise manner compared with the alginate impressions. A previous study found that approximately $0.15-0.5 \%$ of shrinkage occurs in silicone rubber materials following polymerization ${ }^{19)}$. Another study reported that expansion due to water absorption, shrinkage due to water evaporation, and volume reduction due to release of internal liquid by the gel are factors influencing the stability of the dimensions of alginic acid. Furthermore, the authors found that the initial procedure is more dependent on the expanding behavior and that lyotropicity is influenced by the innate components of alginic acid ${ }^{3)}$.

Regarding the data obtained from the three studied regions, the CS had a narrower range of error than the OS in both impression types. Substance shape is one of the factors determining the quality of the scanning data. In accordance with the laser scanner method, Rudolph et $a l .{ }^{20)}$ obtained data from locations known as the light source and the distance angle when the laser beams reaches a 3-D shape; therefore, the positive structure abutment tooth model could scan all of the parts evenly, turning with the movement of the table. However, when a negative structure such as an impression is scanned, it is difficult for the light to reach the deep parts (e.g. the cusp of molar) because they are narrow and shaded. For this reason, the part that is not fully scanned has a small number of point clouds and is different from the original shape.

Although the American Dental Association (ADA) specification no. 18 on dental alginate impression materials does not specify the maximum allowable rate of dimensional change ${ }^{21)}$, ADA specification no. 19 states that the maximum allowable rate of dimensional change in rubber impression materials is $0.40 \%$ for polysulfides and $0.60 \%$ for silicones ${ }^{22}$. The range of the dimensional change rate for the impressions used in this study was between $-0.6 \%$ and $0.55 \%$ (Table 3 ) and therefore in accordance with the parametric variables determined in ADA specification no. 19.

Currently, the majority of clinicians do not use alginate to prepare fixed dental prostheses; this is most likely due to the time-dependent dimensional changes of the alginate impressions. However, a previous study reported that a model prepared with extended- pour alginate impression material did not exceed the parametric variables determined by the ADA within a 5-day duration, and that the conventional alginate impression supported preparation of a stone model within a 2-day duration ${ }^{3)}$. Digitization of alginate impressions, made possible by the introduction of the CAD/CAM system, may have an economic impact because it reduces the required time and cost by omitting the stone model preparation step, and increases the work effectiveness by omitting one step in the conventional fixed prostheses preparation procedure. Furthermore, the efficiency of the procedure and generation of stable data can be improved by the use of alginate impression materials which reinforce dimensional stability.

\section{CONCLUSION}

Results of this study showed that rubber impressions had greater accuracy than alginate impressions. However, these results also carefully suggest the possibility of digitization of alginate impressions in the future. This is because the error was within the maximum allowable tolerance for dimension changes of rubber impressions (material of No. 19 ADA standard) when the digital data of alginate impressions was compared with CRM.

\section{ACKNOWLEDGMENT}

This work was supported by Korea University Grant (K1508381).

\section{REFERENCES}

1) Alcan T, Ceylanoğlu C, Baysal B. The relationship between digital model accuracy and time dependent deformation of alginate impressions. Angle Orthod 2009; 79: 30-36.

2) Murata H, Kawamura M, Hamada T, Chimori H, Nikawa H. Physical properties and compatibility with dental stones of current alginate impression materials. J Oral Rehabil 2004; 31: 1115-1122.

3) Imbery TA, Nehring J, Janus C, Moon PC. Accuracy and dimensional stability of extended-pour and conventional alginate impression materials. J Am Dent Assoc 2010; 141: 32-39.

4) Ceyhan JA, Johnson GH, Lepe X, Phillips KM. A clinical study comparing the three-dimensional accuracy of a working die generated from two dual-arch trays and a complete-arch custom tray. J Prosthet Dent 2003; 90: 228-234.

5) Motohashi N, Kuroda T. A 3D computer-aided design system applied to diagnosis and treatment planning in orthodontics and orthognathic surgery. Eur J Orthod 1999; 21: 263-274.

6) Sinobad T, Obradović-Đuričić K, Nikolić Z, Dodić S, Lazić V, Sinobad V, Jesenko-Rokvić A. The effect of disinfectants on dimensional stability of addition and condensation silicone impressions. Vojnosanit Pregl 2014; 71: 251-258.

7) Wan-Son L, Woong-Chul K, Hea-Young K, Wook-Tae K, Ji-Hwan K. Evaluation of different approaches for using a laser scanner in digitization of dental impressions. J Adv Prosthodont 2014; 6: 22-29.

8) Mehl A, Gloger W, Kunzelmann KH, Hickel R. A new optical 3-D device for the detection of wear. J Dent Res 1997; 76: 1799-1807.

9) Shah S, Sundaram G, Bartlett D, Sherriff M. The use of a 3D laser scanner using superimpositional software to assess the 
accuracy of impression techniques. J Dent 2004; 32: 653-658.

10) Tinschert J, Natt G, Mautsch W, Spiekermann H, Anusavice KJ. Marginal fit of alumina-and zirconia-based fixed partial dentures produced by a CAD/CAM system. Oper Dent 2000; 26: 367-374.

11) DeLong R, Pintado MR, Ko CC, Jodges JS, Douglas WH. Factors influencing optical 3D scanning of vinyl polysiloxane impression materials. J Prosthodont 2001; 10: 78-85.

12) Schaefer O, Schmidt M, Goebel R, Kuepper H. Qualitative and quantitative three-dimensional accuracy of a single tooth captured by elastomeric impression materials: an in vitro study. J Prosthet Dent 2012; 108: 165-172.

13) Quaas S, Rudolph H, Luthardt RG. Direct mechanical data acquisition of dental impressions for the manufacturing of CAD/CAM restorations. J Dent 2007; 35: 903-908.

14) Kamegawa M, Nakamura M, Rukui Y, Tsutsumi S, Jojo M. Direct 3-D morphological measurements of silicone rubber impression using micro-focus X-ray CT. Dent Mater J 2010; 29: 68-74.

15) DeLong R, Heinzen M, Jodges JS, Ko CC, Douglas WH. Accuracy of a system for creating $3 \mathrm{D}$ computer models of dental arches. J Dent Res 2003; 82: 438-442.

16) Persson AS, Oden A, Andersson M, Sandborgh-Enqlund G. Digitization of simulated clinical dental impressions: virtual three-dimensional analysis of exactness. Dent Mater 2009; 25: 929-936

17) Flügge TV, Schlager S, Nelson K, Nahles S, Metzqer MC. Precision of intraoral digital dental impressions with iTero and extraoral digitization with the iTero and a model scanner. Am J Orthod Dentofacial Orthop 2013; 144: 471-478.

18) Trifkovic B, Budak I, Todorovic A, Vukelic D, Lazic V, Puskar T. Comparative analysis on measuring performances of dental intraoral and extraoral optical 3D digitization systems. Measurement 2014; 47: 45-53.

19) Craig RG, Powers JM. Restorative dental materials, 10th ed. St Louis, MO, USA: Mosby, 2002; p. 332-340.

20) Rudolph H, Quaas S, Luthardt RG. Matching point clouds: limits and possibilities. Int J Comput Dent 2001; 5: 155-164.

21) No, A.S., for alginate impression material. Revised ANSI/ ADA Specification, 18-1992.

22) Association, A.D., Guide to Dental Materials and Devices (ed 7) American Dental Association. Chicago 1975; p. 219-229. 